\title{
Rare Features of Dermatomyositis in a Patient with Ovarian Cancer
}

\author{
Lissandra Dal Lago ${ }^{1}$, Frédéric Vandergheynst ${ }^{2}$, Ahmad Awada $^{1}$, Thierry Pepersack $^{1}$ \\ ${ }^{1}$ Medical Oncology Department, Institut Jules Bordet, Université Libre de Bruxelles, Brussels, Belgium \\ ${ }^{2}$ Internal Medicine Department, Cliniques Universitaires de Bruxelles, Hôpital Erasme, Université Libre de Bruxelles, Brussels, Belgium
}

Received: $13 / 08 / 2020$

Accepted: 01/10/2020

Published: $15 / 12 / 2020$

How to cite this article: Da Lago L, Vandergheynst F, Awada A, Pepersack T. Rare features of dematomyositis in a patient with ovarian cancer. EJCRIM 2020;7: doi:10.12890/2020_001946.

Conflicts of Interests: The Authors declare that there are no competing interests.

Acknowledgements: We thank Ms Karen Pickett for her careful proofreading of the manuscript.

This article is licensed under a Commons Attribution Non-Commercial 4.0 License

\section{ABSTRACT}

Despite having a classic presentation of dermatomyositis, a patient with ovarian cancer demonstrated several uncommon features: (i) unexpected onset of dermatomyositis in spite of cancer remission, (ii) development of Evans' syndrome and subcutaneous oedema, and (iii) dysphagia. We discuss the occurrence of these conditions as well as their treatment.

\section{LEARNING POINTS}

- This case illustrates a mode of onset of dermatomyositis that could challenge its classification as a 'paraneoplastic' syndrome, as the dermatomyositis appeared when the patient was in complete metabolic remission.

- This case also raises questions about the observed relationship between IVIG administration and the onset of subcutaneous oedema and Evans' syndrome.

\section{KEYWORDS}

Paraneoplastic syndrome, dermatomyositis, Evans' syndrome, ovarian neoplasm

\section{INTRODUCTION}

Cutaneous paraneoplastic syndrome is poorly understood ${ }^{[1]}$. In most cases, the production of cytokines or other mediators by the tumour is believed to directly or indirectly cause the condition. Confusion also remains as to what constitutes a 'paraneoplastic' sign or symptom. Etymologically, the prefix 'para' denotes 'alongside', so paraneoplastic suggests an event occurring at the same time as a malignancy; the onset of symptoms during complete remission is uncommon.

\section{CASE DESCRIPTION}

A 74-year-old woman was admitted from a cancer centre to the geriatric oncology clinic on 10 January 2019 because of an erythematous skin eruption. She had a history of arterial hypertension, thyrotoxicosis and epilepsy. In 2014, she had been diagnosed with serous ovarian cancer with splenic, liver and peritoneal metastases (stage IIIc) and had been treated with success with three neoadjuvant cycles of carboplatinpaclitaxel followed by internal debulking surgery and adjuvant chemotherapy (cisplatin-cyclophosphamide). In July 2018, her condition had relapsed with new splenic, liver and peritoneal metastases and she had been treated with three cycles of carboplatin-gemcitabine, resulting in complete metabolic remission documented by positron emission tomography-computed tomography (PET-CT). In November 2018, a further relapse (new splenic, liver, and peritoneal metastases) was treated with three cycles of gemcitabine (final dose on 27 December 2018). 
On admission in January 2019, skin examination of the patient showed a heliotropic rash on both eyelids, a 'V' sign on her neck, Gottron's papules on the backs of both hands and periungual erythema, suggesting a diagnosis of dermatomyositis. There was no functional decline or muscle weakness and the creatine kinase (CK) concentration was within the normal range.

However, within 5 days, the patient's condition deteriorated rapidly and she became bedridden. Blood test results showed elevated serum concentrations of CK (16,027 IU/I; normal values < $167 \mathrm{IU} / \mathrm{I})$ and C-reactive protein (CRP; $94 \mathrm{mg} / \mathrm{I})$. Her white blood cell count was 4,140/ $\mu$ l, glomerular filtration rate $79 \mathrm{ml} / \mathrm{min}$, creatinine $0.75 \mathrm{mg} / \mathrm{dl}$, urea $18 \mathrm{mg} / \mathrm{dl}$, glutamic-oxaloacetic transaminase 314 IU/I, glutamic-pyruvic transaminase $100 \mathrm{IU} / \mathrm{I}$, alkaline phosphatase $48 \mathrm{IU} / \mathrm{I}$, gamma glutamyltransferase $47 \mathrm{IU} / \mathrm{l}$, thyroid stimulating hormone $1.80 \mathrm{mU} / \mathrm{l}$, and free-T4 $16.2 \mathrm{pmol} / \mathrm{l}$. She had a positive antinuclear antibody test (1:1280) with elevated titres of anti-Sjögren's syndrome-related antigen A (anti-SSA 52 (RO52)) and anti-transcriptional intermediary factor (TIF)-1y. Muscle biopsy showed diffuse inflammatory cells in the connective tissue and more focally in the endomysium. Whole body $18 \mathrm{~F}$-fluorodeoxyglucose PET confirmed complete metabolic remission of the ovarian tumour.

On day 0 , treatment with $1 \mathrm{mg} / \mathrm{kg} /$ day oral methylprednisolone was started, but the patient developed severe dysphagia after 10 days, so we administered intravenous pulses of methylprednisolone $500 \mathrm{mg} /$ day for 3 days, followed by a tapering dose of oral prednisolone, and $0.4 \mathrm{mg} /$ kg intravenous immunoglobulin (IVIG) for 4 days (Fig. 1). The patient also received enteral nutrition. One week later, the patient developed haemolytic anaemia (Coombs positive) associated with unexplained thrombocytopenia, and serum CK concentrations, which had been decreasing, started to rise again. We also observed marked subcutaneous oedema (the serum albumin concentration was within the normal range). Rituximab was prescribed which was associated with progressive normalization of biochemical abnormalities and improvement in the patient's functional status. She was discharged after 8 weeks, and had completely recovered at follow-up 2 months later.

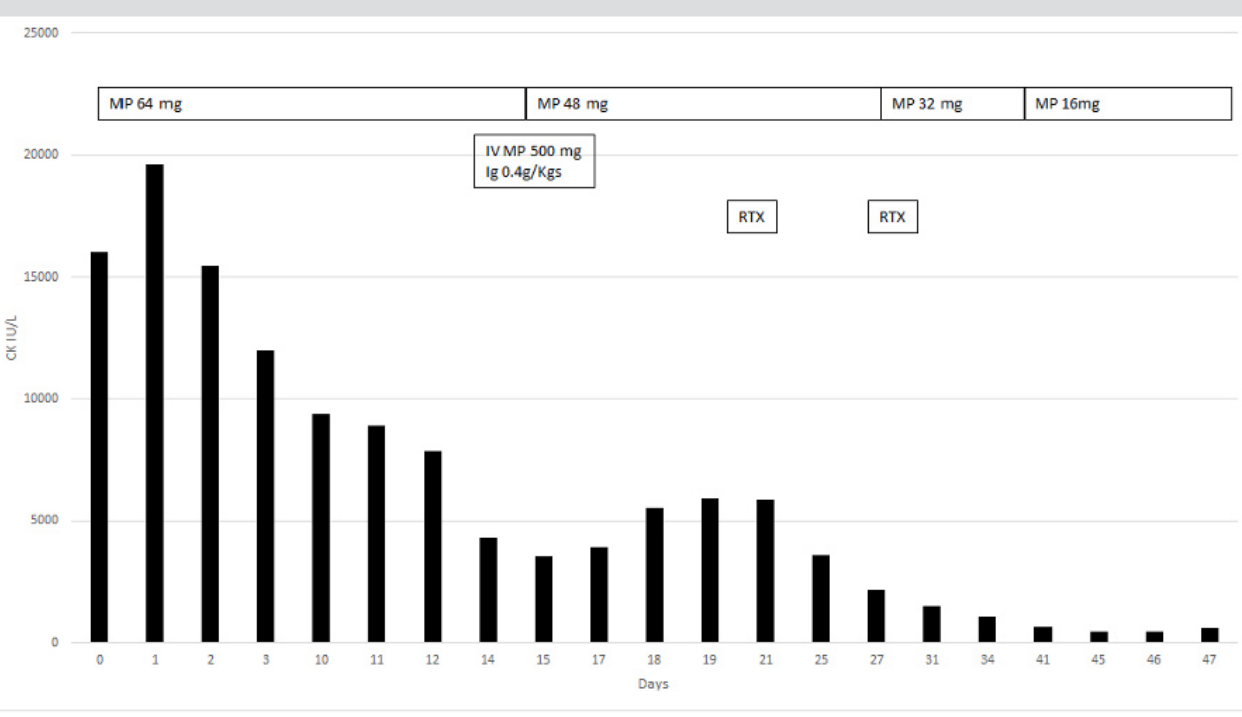

Figure 1. Evolution of serum creatine kinase (CK) concentrations and treatment administration CK: serum creatinine kinase; MP: methylprednisolone; Ig: immunoglobulin, RTX: rituximab.

\section{DISCUSSION}

Despite an initial classic presentation of dermatomyositis, this patient had the following, uncommon clinical features: (i) unexpected onset in a patient with complete metabolic tumour remission, (ii) Evans' syndrome and subcutaneous oedema, (iii) and dysphagia.

\section{Unexpected onset of dermatomyositis}

In this patient, dermatomyositis developed despite a complete metabolic response to cancer chemotherapy and without any other incidental primary malignancy. The fact that the symptoms appeared despite a good response to chemotherapy raises a semantic issue as to whether this condition can be called 'paraneoplastic'. Dermatomyositis syndrome has previously been described in patients with advanced ovarian cancer, including after recurrence ${ }^{[1]}$. We hypothesize that the tumour lysis induced by chemotherapy may trigger an immune response (neoantigen exposure) leading to dermatomyositis. Also, even in the absence of metabolic relapse during observation of our case, we cannot rule out that the patient did not present residual cancer activity. If we accept that a paraneoplastic syndrome could appear during the preclinical stage of a cancer, we could probably also accept that the same condition could occur during clinical remission. 
Subcutaneous oedema and Evans' syndrome

Although periorbital oedema is a common manifestation of dermatomyositis, generalized subcutaneous oedema is extremely rare in this setting and its exact mechanism is not clear. Cathepsin G (CTSG) is a member of the serine protease family and can increase the permeability of vascular endothelial cells and chemotaxis of inflammatory cells. Gao et al. ${ }^{[2]}$ reported that the expression of CTSG was increased in the peripheral blood mononuclear cells and muscle tissues of patients with dermatomyositis and correlated with disease activity. Serum CTSG induced expression of protease activated receptor 2 (PAR2) and altered the cytoskeleton of human dermal microvascular endothelial cells, thus potentially playing a role in muscle inflammatory cell infiltration by increasing vascular endothelial cell permeability.

Evans' syndrome is rarely a presenting feature of dermatomyositis ${ }^{[3,4]}$. In our patient, the onset of Evans' syndrome after the administration of IVIG was quite unexpected because IVIG has been proposed as a treatment for this condition ${ }^{[5]}$. We hypothesize that Evans' syndrome was refractory to IVIG in our patient.

\section{Dysphagia}

This case illustrates well the described association between dysphagia and the presence of anti-TIF-1 $y$ antibodies ${ }^{[6,7]}$. The presence of dysphagia is known to be associated with a worse prognosis in patients with dermatomyositis ${ }^{[8,9]}$. In a patient with severe myopathy affecting bulbar muscle groups, an infusion of IVIG was associated with improvement in the steroid-refractory myopathy ${ }^{[10]}$. However, when additional muscle weakness develops during corticosteroid treatment of an inflammatory myopathy, it is important to distinguish between an exacerbation of the myopathy and glucocorticoid-induced myopathy ${ }^{[11]}$, including the very exceptional onset of isolated laryngopharyngeal steroid myopathy. In the present case, it is likely that the dysphagia was a result of inflammatory dermatomyositis rather than glucocorticoid toxicity because of the early onset of dysphagia and the high CK concentration.

\section{REFERENCES}

1. Flynn M, Ottaway Z, Kaur J, Waters J. Three differently timed presentations of dermatomyositis associated with advanced ovarian cancer. BMJ Case Rep 2018;2018:bcr2017222627.

2. Gao S, Zhu H, Yang H, Zhang H, Li Q, Luo H. The role and mechanism of cathepsin G in dermatomyositis. Biomed Pharmacother 2017;94:697-704.

3. Hay EM, Makris M, Winfield J, Winfield DA. Evans' syndrome associated with dermatomyositis. Ann Rheum Dis 1990;49(10):793-794.

4. Flores-Terry MA, Garcia-Arpa M, Anino-Fernandez J, Minguez-Sanchez MD. Edematous dermatomyositis with probable Evans syndrome. Actas Dermosifliogr 2017;108(7):673675.

5. Chang DK, Yoo DH, Kim TH, Jun JB, Lee IH, Bae SC, et al. Induction of remission with intravenous immunoglobulin and cyclophosphamide in steroid-resistant Evans' syndrome associated with dermatomyositis. Clin Rheumatol 2001;20(1):63-66.

6. Kurushima S, Horai Y, Umeda M, Kawakami A. Anti-transcription intermediary factor1gamma-antibody-positive dermatomyositis complicated by dysphagia. Intern Med 2017;56(21):2965-2966

7. Mugii N, Hasegawa M, Matsushita T, Hamaguchi Y, Oohata S, Okita H, et al. Oropharyngeal dysphagia in dermatomyositis: associations with clinical and laboratory features including autoantibodies. PLoS One 2016;11(5):e0154746.

8. Ogawa-Momohara M, Muro Y, Kono M, Akiyama M. Prognosis of dysphagia in dermatomyositis. Clin Exp Rheumatol 2019;37(1):165.

9. Kwon KM, Lee JS, Kim YH. A case report of life-threatening acute dysphagia in dermatomyositis: challenges in diagnosis and treatment. Medicine (Baltimore) 2018;97(17):e0508.

10. Hafejee A, Coulson IH. Dysphagia in dermatomyositis secondary to bladder cancer: rapid response to combined immunoglobulin and methylprednisolone. Clin Exp Dermatol 2005;30(1):93-94.

11. Pereira RM, Freire de Carvalho J. Glucocorticoid-induced myopathy. Joint Bone Spine 2011;78(1):41-44 